\title{
Grapevine Resistance to the Nematode Xiphinema index Is Durable in Muscadine-Derived Plants Obtained from Hardwood Cuttings but Not from In Vitro
}

\author{
Van Chung Nguyen,, ${ }^{1}$ Jean-Pascal Tandonnet, ${ }^{2}$ Samira Khallouk, ${ }^{3}$ Cyril Van Ghelder, ${ }^{1}$ Ulysse Portier, ${ }^{1}$ Maria Lafargue, ${ }^{2}$ \\ Mohamed Youssef Banora, ${ }^{4}$ Nathalie Ollat, ${ }^{2}$ and Daniel Esmenjaud ${ }^{1, \dagger}$ \\ ${ }^{1}$ INRAE, Université Côte d'Azur, CNRS, ISA, 06903 Sophia Antipolis, France \\ 2 University of Bordeaux, Bordeaux Sciences Agro, INRAE, ISVV, EGFV, 33882 Villenave d'Ornon, France \\ ${ }^{3}$ Service de la Protection du Patrimoine Végétal, ONSSA, Rabat, Morocco \\ ${ }^{4}$ Ain Shams University, 11241 Cairo, Egypt \\ Accepted for publication 4 May 2020.
}

\begin{abstract}
Breeding for varieties carrying natural resistance $(\mathrm{R})$ against plantparasitic nematodes is a promising alternative to nematicide ban. In perennial crops, the long plant-nematode interaction increases the risk for $\mathrm{R}$ breaking and $\mathrm{R}$ durability is a real challenge. In grapevine, the nematode Xiphinema index has a high economic impact by transmitting Grapevine fanleaf virus (GFLV) and, to delay GFLV transmission, rootstocks resistant to this vector are being selected, using Muscadinia rotundifolia in particular as an $\mathrm{R}$ source. To optimize in fine this strategy, the durability has been studied under controlled conditions in F1 and BC1 muscadine-derived resistant accessions previously obtained from either hardwood-cutting or in vitro propagation. After inoculation with a mix, in

reproduction factor remained lower than 1 in resistant plants obtained from hardwood cuttings while it increased at values far beyond 1 in resistant plants of in vitro origin. Data for nematode numbers per gram of roots mostly paralleled those obtained for the reproduction factor. The effect of the propagation type on resistance over years was also evaluated for the ratio female/juvenile and the frequency of males. Altogether our results illustrate that the muscadine-derived resistance based on hardwood cuttings is durable. By contrast, in resistant and reference accessions obtained from in vitro, our data suggest that the increased nematode multiplication might be mainly due to the modification of root architecture consecutive to this propagation method.
\end{abstract} equal proportions, of four lines representative of the $X$. index diversity, multiplication on plants has been monitored 3 to 6 years. The nematode

The nematode Xiphinema index Thorne and Allen, 1951 (Dorylaimida, Longidoridae) is present in all major grapegrowing regions and particularly in Europe, the Mediterranean Basin, North and South America, and the Middle East from where it originates (Nguyen et al. 2019). This dagger nematode has a high economic impact in grapevine by transmitting Grapevine fanleaf virus (GFLV), the major virus of this crop at the world scale (Andret-Link et al. 2004; Jones et al. 2013). Control of $X$. index was mainly based on chemical nematicides that are progressively banned in most developed countries because of their high acute toxicity to humans and environment. Control alternatives such as fallow crops (Villate 2008; Villate et al. 2012) and natural resistance are being studied.

Breeding rootstocks with natural resistance to $X$. index appears particularly promising as grapevines are grown on rootstocks that are genetically different from the variety and can be selected

†Corresponding author: D. Esmenjaud; daniel.esmenjaud@inrae.fr

Current address for V. C. Nguyen: Plant Pathology Group, Plant Protection Research Institute (PPRI), Hanoï, Vietnam.

Funding: This study was partly funded by the French Ministry of Agriculture through the CASDAR contracts 'RemuneX' (2015-2018) (CTPS Project number C-2014-09). Complementary funding was provided by the contracts INRAAgriObtentions IVD3 (2011-2015) and IVD4 (2016-2020).

*The $\boldsymbol{e}$-Xtra logo stands for "electronic extra" and indicates there are supplementary materials published online.

The author(s) declare no conflict of interest.

(C) 2020 The American Phytopathological Society
Keywords: genetics and resistance, nematology independently, provided that they still allow graft compatibility. Resistance to $X$. index has been studied in California and France (Esmenjaud and Bouquet 2009; Esmenjaud et al. 2010). It has been searched for in Vitis spp. and in the close genus Muscadinia. Some clonal accessions of $V$. riparia and $V$. arizonica express a higher resistance level than other Vitis spp. and an R quantitative trait locus (QTL) located on chromosome 19 (XiRl) has been evidenced in this later species (Hwang et al. 2010). Nevertheless, the highest resistance level has been observed in some muscadine (M. rotundifolia) accessions (Boubals and Pistre 1978). Despite the low fertility of the Vitis-Muscadinia crosses due to the differences in basic chromosomal numbers between the two genera $(2 n=38$ in Vitis; $2 n=40$ in Muscadinia), a few intergeneric $\mathrm{F} 1$ and $\mathrm{BC} 1$ accessions were created in France and some of them have shown a high level of resistance to the nematode and a significant cognate delay in GFLV infection. Natural resistance to the vector might act as a barrier or at least a filter to viral transmission (Bouquet et al. 2000). In particular, the F1 individuals VRH97-99-79 (muscadine source Carlos) or VRH8771 (muscadine source NC184-4) significantly reduced nematode numbers (Esmenjaud et al. 2010). The BC1 resistant individual RPG1 (source NC184-4) (Esmenjaud et al. 2010) showed delayed GFLV infection in the vineyard (Ollat et al. 2011), which justified its registration as a rootstock called 'Nemadex Alain Bouquet'. In plants derived from the muscadine source NC184-4, a polygenic resistance conferred by three dominant QTLs (XiR2 to XiR4) located respectively on chromosomes 9, 10, and 18 (Rubio et al. 2020) has been characterized and $X$. index induces an hypersensitive-like response (D. Esmenjaud and M. Banora, unpublished data).

In grapevine, rootstocks are classically reproduced by vegetative propagation from hardwood cuttings. An alternative vegetative 
multiplication is in vitro culture, a method more commonly used for stock conservation. This propagation type may influence the expression of natural resistance to pests and diseases. Evidence of the effect of in vitro culture in susceptibility to pathogens has been shown early (Nozeran 1978). In sugarcane rust (Puccinia melanocephala) disease, susceptibility to the fungus depends on the number of in vitro culture cycles (Péros et al. 1994). Grapevine (Grenan 1982) as well as several species of in vitro-cultured plants (Nozeran 1980) have been shown to express juvenile characteristics that modify their interaction with pathogens. In nematodes, preliminary data obtained by Esmenjaud et al. (2010) suggested that $X$. index adapted progressively to resistant muscadine-derived accessions obtained from in vitro plants with an increase in reproduction over 4 years after inoculation.

In this manuscript, we report a durability study that has considered resistance to $X$. index in $\mathrm{F} 1$ and $\mathrm{BC} 1$ representative accessions both derived from the muscadine source NC184-4. The effect of the propagation type has been evaluated using plants previously obtained from either hardwood-cutting or in vitro propagation. In our study, inoculum was a combination of four isofemale lines representative of the nematode worldwide diversity (Nguyen et al. 2019). Our results have monitored the nematode multiplication yearly in plants from each type between 3 and 6 years. We have shown that the resistance durability evaluated from various nematode developmental criteria depends on the propagation type.

\section{MATERIALS AND METHODS}

Creation and characterization of $X$. index lines. Single female descendants or isofemale lines (referred hereafter as lines) were created from single individuals inoculated in $100-\mathrm{ml}$ pots containing fig plantlets (Ficus carica 'Goutte d'or') previously obtained from in vitro. Four final lines were selected on the basis of their $C y t B$ mitochondrial haplotype and their microsatellite genotype (Villate et al. 2009; Nguyen 2018) and, after extensive multiplication onto fig containers, were used as inoculum. These lines originated from (West to East): Spain ('La Mancha'; Western Mediterranean), Italy ('Pallo del Colle'; Central Mediterranean), Greece ('Samos'; Eastern Mediterranean), and Iran ('Sharekord'; Middle East). Nguyen et al. (2019) showed that these lineages belong to three of the four subclades revealed in an $X$. index worldwide phylogeographical study.

Plant accessions. We used two resistant $(\mathrm{R})$ clonal accessions derived from the muscadine source NC184-4. These were the F1 accession 'VRH8771' (8771), carrying the three resistance QTLs
(XiR2 to XiR4), and the BC1 accession 'Nemadex Alain Bouquet' (RPG1) (Fig. 1). The accession V. vinifera 'Cabernet-Sauvignon' (CS) was the susceptible (S) reference (Fig. 1). The rootstock V. riparia 'Gloire de Montpellier' (RGM) was used as a reference accession expressing an intermediate (I) phenotype. For each accession, two types of plants have been evaluated: plants previously rooted as hardwood cuttings (hardwood-cutting) and plants acclimatized in soil from in vitro plantlets (in vitro) (Supplementary Table S1).

In vitro plants. Plants obtained from in vitro originated from in vitro cultures first established in the year 2000. Plants have been maintained at $20^{\circ} \mathrm{C}$ with a $16 \mathrm{~h}$ photoperiod on multiplication (MM) and rooting media (RM) (Supplementary Table S2) derived from the MS medium (Murashige and Skoog 1962). Every 2 months, new pieces of shoots have been cut from the old culture and planted for new culture onto MM for conservation purpose (without plant hormones). They have then been kept for several rounds as MM cultures until their rooting onto RM (= MM supplemented with indole-3-butyric acid (IBA) at $0.5 \mathrm{mg} / \mathrm{liter}$ ) for their subsequent acclimatization and use in the durability experiment. After 6 weeks, rooted plantlets were transferred into trays filled with a perlite substrate without hormones in an acclimatization chamber at $24^{\circ} \mathrm{C}$ for 6 weeks. Plants were then moved to the greenhouse, transplanted individually into 2-liter containers filled with a sterile sand-loam soil, and inoculated 2 months later (Fig. 2). Two months after inoculation, plants were gently repotted into 4-liter containers. Consequently, the delay between the beginning of the acclimatization of plantlets (end of the in vitro steps) and their inoculation has been approximately 3 to 4 months (Fig. 2).

Hardwood-cutting plants. These plants were sampled in spring from dormant grapevine mother plants in the repository. Hardwood cuttings with two nodes were planted individually for rooting into $150-\mathrm{ml}$ cells filled with a fine-sand substrate. No rooting or shooting hormones were used. Plants were placed onto benches and regularly humidified with a mist system. After 8 weeks, rooted hardwood cuttings were repotted into 2 -liter pots filled with a sterile sand-loam soil. Plants were inoculated after 2 months and gently repotted into 4-liter containers after 2 more months (Fig. 2).

Inoculation method and experimental design. Nematodes were extracted from soil using the elutriation method adapted from Oostenbrink (Hooper 1986; Van Ghelder et al. 2015). Inoculations were performed using a total of 200 individuals comprising 50 individuals from each of the four lines. The individuals were suspended in water and deposited into two holes, 2-cm deep, and $2 \mathrm{~cm}$ from the stem.

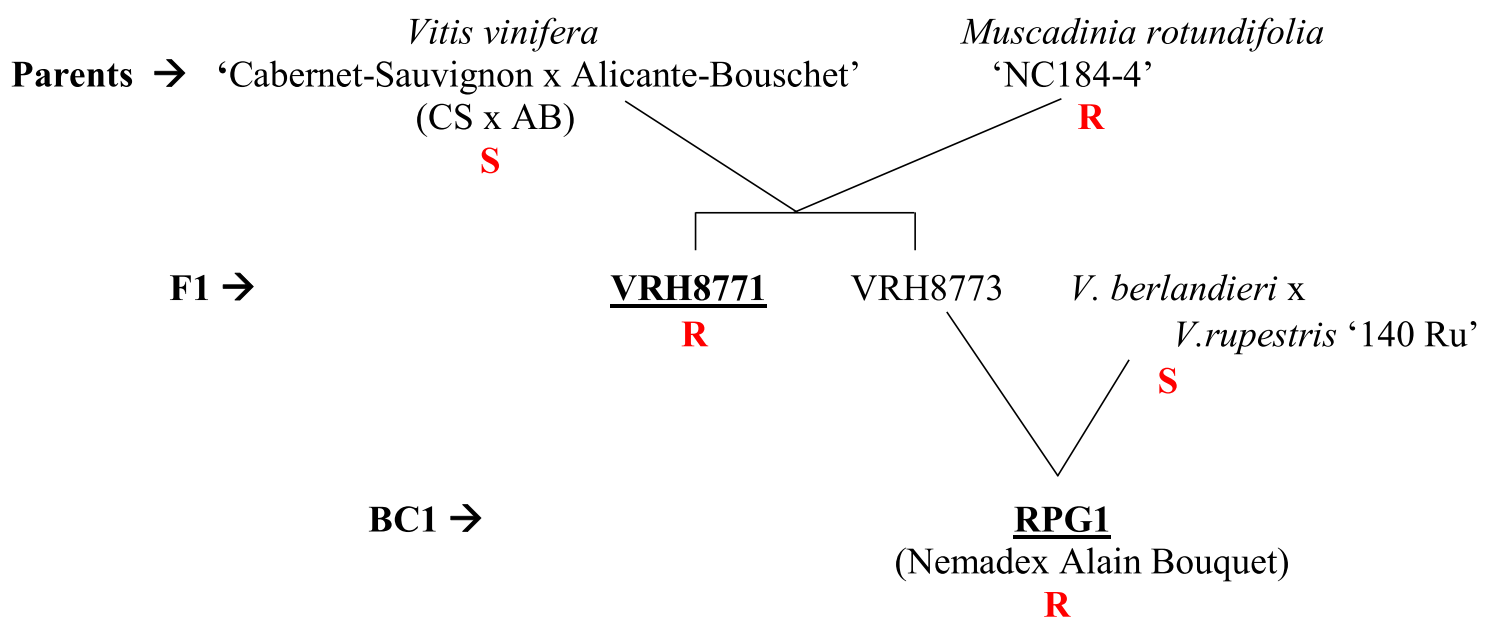

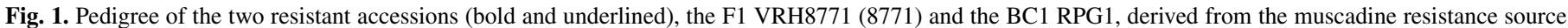
NC 184-4. Cabernet-Sauvignon (CS) is the susceptible reference accession used in the study. R, resistant; S, susceptible. 
In our experimental design, plants obtained from in vitro and plants obtained from cuttings have been installed on adjacent benches in the greenhouse. Nevertheless, plant growth conditions were considered equivalent as plants occupied benches positioned centrally with similar climatic conditions in the greenhouse. Within each propagation type, all plants (whatever their accession and sampling year) have been completely randomized. In each propagation type, two plants from each clone were also added randomly as noninoculated controls.

The protocol has been set up for a total duration of 6 years using the four clonal accessions (RPG1, 8771, RGM, and CS) grown from either hardwood cuttings or in vitro (Fig. 2, Supplementary Table S1). Containers were drip irrigated daily and temperature was regulated to reach a maximum of $30^{\circ} \mathrm{C}$. To avoid winter frost, the greenhouse was heated to keep temperatures above $5^{\circ} \mathrm{C}$. As low temperatures permitted winter leaf fall, plants were pruned every year after it by keeping two eyes on each major shoot.

Criteria of resistance evaluation. At each sampling date (in 3-, 4-, 5-, and 6-year-old plant pots), the aerial part of each plant was cut at the collar and each container was hermitically placed into a double plastic bag and transferred into a climatic chamber at $6^{\circ} \mathrm{C}$ until soil nematode extraction. Total nematode numbers (J1 to J4 juveniles, female adults, and male adults) were evaluated from the entire soil volume of each container. Resistance was evaluated primarily by the criterion reproduction factor $(\mathrm{RF})$ of the nematode, i.e., the ratio between total numbers evaluated at a given plant age and the initial inoculum (200 individuals). This ratio was calculated for each container (replicate) and mean of all replicates was used to evaluate the RF for each accession. Accessions were classified as resistant (R) when their RF was $<1$ and susceptible (S) when their $R F$ was $\geq 1$. Numbers of juveniles, females, and males were counted and allowed to calculate the ratio female/juvenile and the frequency of males. Weight of below-ground part of each plant replicate was also measured to evaluate (total) nematode numbers per gram of roots $(\mathrm{Nb} / \mathrm{gr})$.

Statistical analysis of the data. Plants obtained from hardwood cuttings and plants obtained from in vitro were analyzed separately. In each propagation type, the rating criteria were considered with the model $Y=$ accession + age of plants + interaction term. Data from combinations of the factors "accession" and "plant age" were analyzed by a two-factor analysis of variance (ANOVA) using XLSTAT software (version 2014.5.03; Addinsoft, Paris, France). The effects of the factors "accession," "plant age," and of their interaction "accession-age" were computed at $P \leq 0.05$

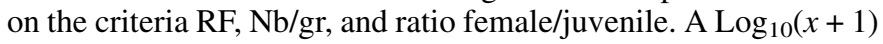
transformation for normal distribution of the criteria $\mathrm{RF}$ and $\mathrm{Nb} / \mathrm{gr}$ was performed before analysis (Noe 1985). Detailed $F$ values and probabilities $P>F$ for each factor and their interaction are reported in Supplementary data (Results section). The combinations accession-age were submitted to a multiple-comparison analysis: statistical differences for the criteria $\log (\mathrm{RF}+1), \log (\mathrm{Nb} / \mathrm{gr}+1)$, and ratio female/juvenile were established using Fisher's least significant difference multiple range test at $P \leq 0.05$.

The effect of the propagation type, i.e., "plants obtained from cuttings" versus "plants obtained from in vitro" was evaluated for each combination "accession-age" using a $t$ test (bilateral) at $P \leq$ 0.05 (XLSTAT software, version 2014.5.03). $P$ values for each combination are reported in Supplementary data (Results section).

\section{RESULTS}

We considered the four clones, 8771 (R), RPG1 (R), RGM (intermediate), and CS (S), obtained either from hardwood cuttings or from in vitro, for analysis over 3 to 6 years after planting. In each propagation type, the RF, the nematode number per gram of roots, and the number of each developmental stage were evaluated.

RF. In hardwood-cutting plants, synthesis of ANOVA data showed that the factor accession was significant, while neither the factor plant age nor the interaction accession-plant age were significant (Supplementary Table S3A). RFs ranged between 0.14 and 0.67 for 8771 and between 0.44 and 0.94 in RPG1 (Fig. 3A). Thus, in these cuttings, RFs always remained under the threshold of 1 that states the plant resistance status. When all years are confounded, RFs were statistically higher in RPG1 than in 8771 (Supplementary Table S3A). As expected for RGM and CS reference accessions (Fig. 3A), values were significantly different from resistant accessions and ranged from 4.0 to 5.16 and from 13.02 to 23.19 , respectively.

By contrast, in in vitro plants, both the factors accession and plant age together with their interaction were significant (Supplementary Table S4A). Over years, RFs displayed high variations among resistant accessions, i.e., between 1.0 and 12.59 in 8771 and between 0.71 and 5.12 in RPG1 (Fig. 3B). RFs of accessions RGM and CS ranged between 7.62 and 33.51 and between 43.02 and 51.83 , respectively.

Comparison of the two propagation types from 4 to 6 years in resistant accessions 8771 and RPG1 showed that RFs for in vitro plants were always significantly greater than in hardwood-cutting plants (Fig. 3 and Supplementary Table S5A). At the first sampling date ( 3 years), the difference was significant in 8771 and not in RPG1 (Fig. 3 and Supplementary Table S5A). In CS and RGM, RFs between propagation types were most often significantly different (Fig. 3A and Supplementary Table S5A).

Nematode number per gram of roots. The total nematode number per gram of roots is a complementary evaluation of the capacities of food supply provided by the plant for the development of $X$. index. In plants obtained from cuttings, ANOVA data showed a significant effect of the factor accession (as previously for the criterion RF) and of the interaction accession-age (Supplementary Table S3B). The resistant accessions 8771 and RPG1 harbored low numbers ranging from 0.6 to 2.2 and from 2.2 to 3.2 individuals per gram of roots, respectively (Fig. 4A). Meanwhile, RGM and CS most often harbored significantly higher numbers, ranging from 6.9 to 14.1 and from 24.3 to 52.7 individuals, respectively (Fig. 4A).

In in vitro plants, ANOVA data showed a significant effect of the factor accession and of the interaction accession-age of plants (Supplementary Table S4B). The resistant accessions had the lowest numbers per gram of roots with 6.6 to 11.9 individuals in RPG1 and

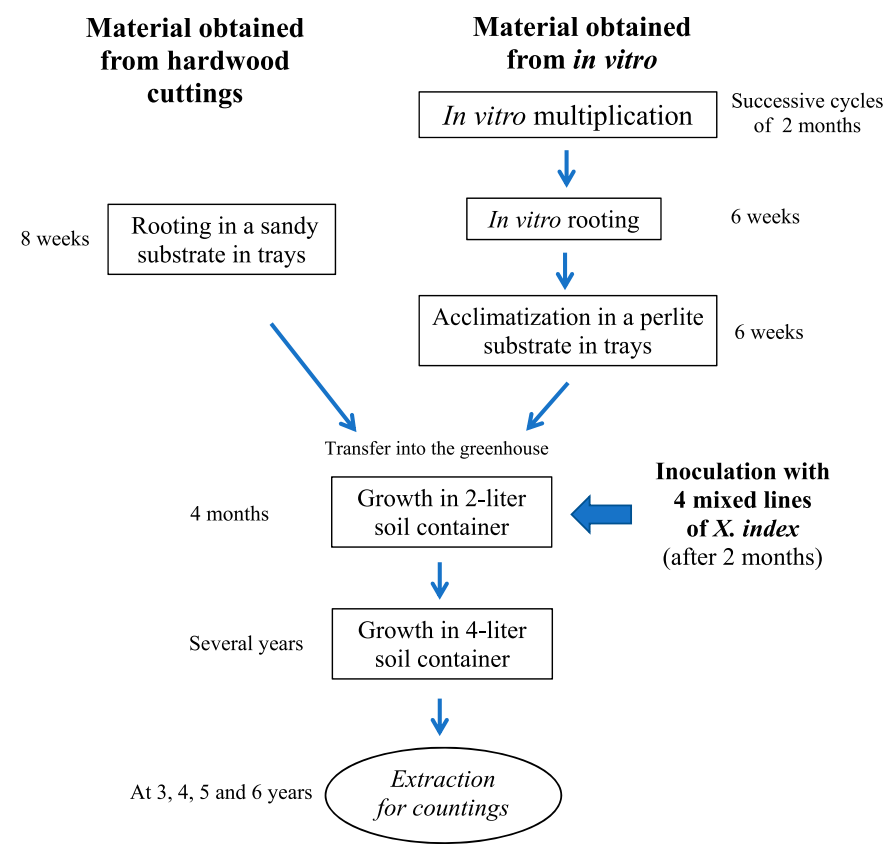

Fig. 2. Steps and timing of the study using plants obtained from hardwood cuttings and from in vitro. 
12.6 to 20.2 individuals in 8771 (Fig. 4B). CS had the highest numbers, i.e., from 322 to 430 nematodes per gram of roots, while RGM had intermediate numbers ranging from 23.6 to 79 individuals (Fig. 4B).

As for the RF criterion from 4 to 6 years, densities for in vitro plants in resistant accessions 8771 and RPG1 were always significantly greater than in hardwood-cutting plants (Fig. 4 and Supplementary Table S5A). Thus, densities in 8771 and RPG1 were, respectively, 6 to 20 times and 3 to 4 times higher in in vitro plants than in hardwood-cutting plants. In CS, this increase was also observed (7 to 13 times) with statistical differences at least nearly significant $(P=0.0548)$. Densities also increased in RGM but to a lesser extent (1.6 to 6 times). Finally, in in vitro plants, results from the number per gram of roots paralleled those from the RF criterion.

Ratio female/juvenile. As males are very rare in $X$. index (see next part), they can be neglected numerically and the ratio

A Cuttings

60

3 years

4 years

5 years

6 years

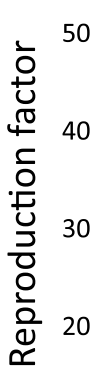

20

10

0

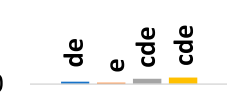

8771

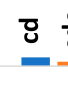

RPG1

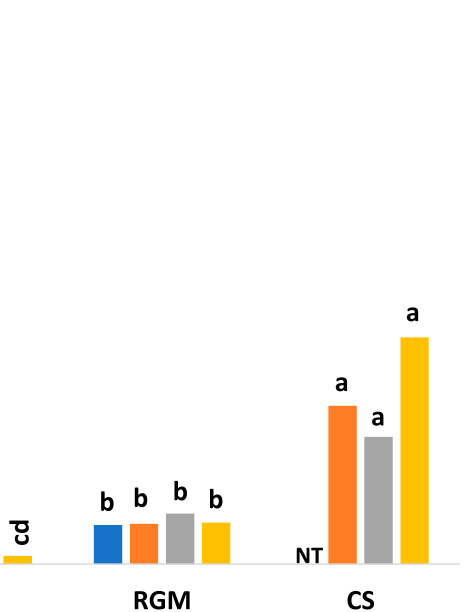

B In vitro

60

50

40

30

20

10

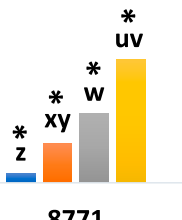

\section{B In vitro}

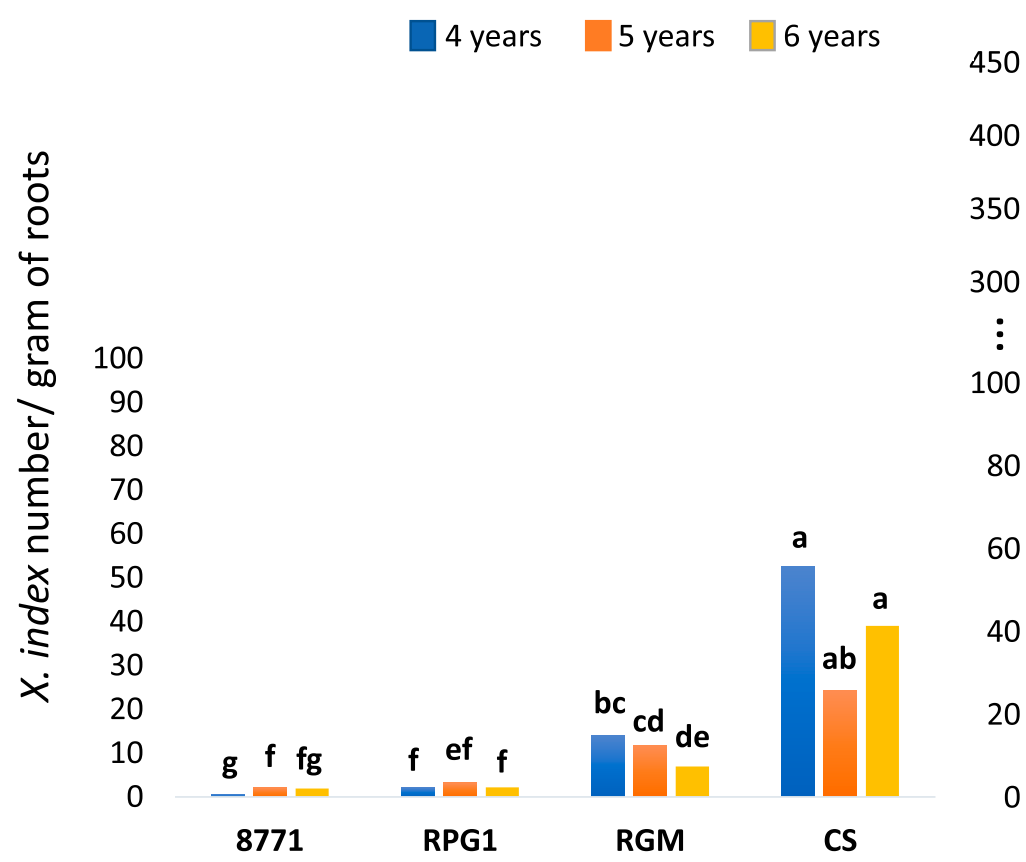

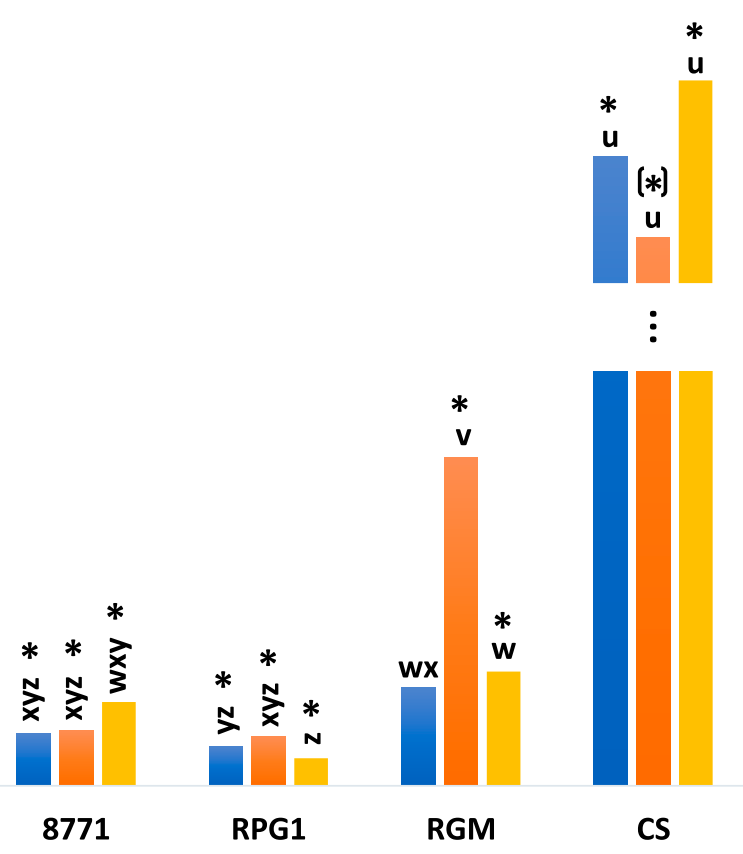

Fig. 3. Reproduction factors to Xiphinema index for accessions 8771 , RPG1, RGM, and CS at 3, 4, 5, and 6 years. A, Cuttings = plants obtained from hardwood

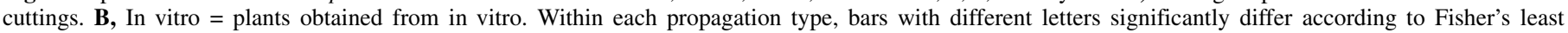

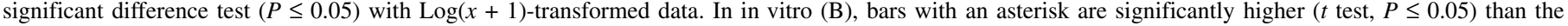

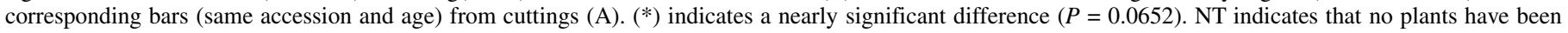
tested at this sampling date.

\section{A Cuttings}

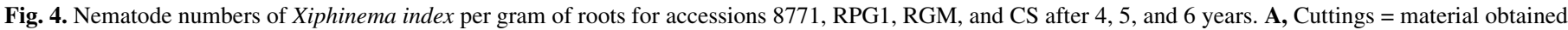

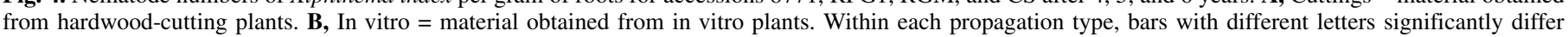

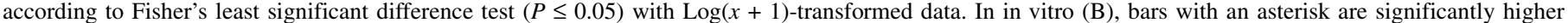
( $t$ test, $P \leq 0.05)$ than the corresponding bars (same accession and age) from cuttings $($ A). $(*)$ indicates a nearly significant difference $(P=0.0548)$. 
female/juvenile may be a good gauge of the status of the nematode population. In hardwood-cutting plants, the factors accession, age, and their interaction were significant for this criterion (Supplementary Table S3C). When all accessions are taken together, 4-year plants had values ranging from 41.0 to $76.8 \%$, while in 5-year plants, the ratio increased in both resistant accessions and remained low in others (Fig. 5A). In 6-year plants, values were very high in $8771(154.3 \%)$ and RPG1 (163.8\%) and also increased in the other accessions to higher values (77.4\% in CS and $93.2 \%$ in RGM) than in previous years (Fig. 5A). These values clearly showed an increased proportion of the female stage in plants aged 5 (8771 and RPG1) and 6 years (8771, RPG1 and RGM). This increase was much more pronounced in the two resistant accessions. As the numbers of individuals recovered in resistant plants have remained low and stable over the years 4 to 6 , these values illustrate that their respective populations have become older, which is in line with the resistant status of their accessions. Even though the phenomenon is less marked, the final increased ratios of females in RGM and CS also suggest that nematode numbers might have reached in these accessions a high and saturating density, which restricts the production of new progenies by the females.

Interestingly, this aging of the nematode population has not been observed in the plants obtained from in vitro. ANOVA analysis showed lower $F$ values and only a significant effect for the factor age (Supplementary Table S4C). In plants from this propagation type, the ratio was relatively stable whatever the age of the plants and ranged from 38.7 to a maximal value of $63.3 \%$ (Fig. 5B). There was no marked difference between resistant plants, i.e., 8771 (44.3 to 59.2) and RPG1 (50.9 to 63.2), versus the intermediate RGM
(38.7 to 59.0) or the susceptible CS (44.1 to 63.3) plants. Our observations are confirmed by the analysis of the effect of the propagation type ( $t$ test) that was significant at 5 years $(8771)$ and 6 years (8771, RPG1 and RGM) (Fig. 5 and Supplementary Table S5B).

Detection and frequency of males. As males are known to occur at very low frequencies in $X$. index, counting males is difficult and subject to misevaluation when dispersed among very high female numbers (i.e., in susceptible and intermediate accessions) and results are more reliable when few nematodes develop (resistant accessions). Consequently, we focused on the resistant accessions and compared male frequencies according to their propagation type. We obtained a range of only zero to two males in total replicates from resistant accessions of a given propagation type at a given age (Table 1). Actually, during the years 4 to 6 , a total number of only 7 males out of approximately 85,000 individuals were picked out, i.e., a mean occurrence of less than 1 male per 10,000 nematodes.

Even though the overall ratio of males was very low, we observed a difference between propagation types as the frequency of males was 5.93 per 10,000 in plants obtained from cuttings while it was only 0.27 per 10,000 , a ratio 20 times lower, in plants obtained from in vitro. Thus, our data suggest a higher frequency in plants obtained from cuttings than from in vitro. Such a difference cannot be validated statistically since (i) too few male individuals were recovered in each propagation type and (ii) samples were totally unbalanced with a total nematode sampling number nine times inferior in plants obtained from cuttings $(8,427$ individuals) than from in vitro $(74,301)$.
A

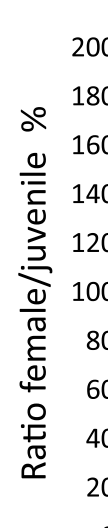$$
\text { Cuttings }
$$$$
4 \text { years }
$$$$
5 \text { years }
$$$$
6 \text { years }
$$

\section{B In vitro}

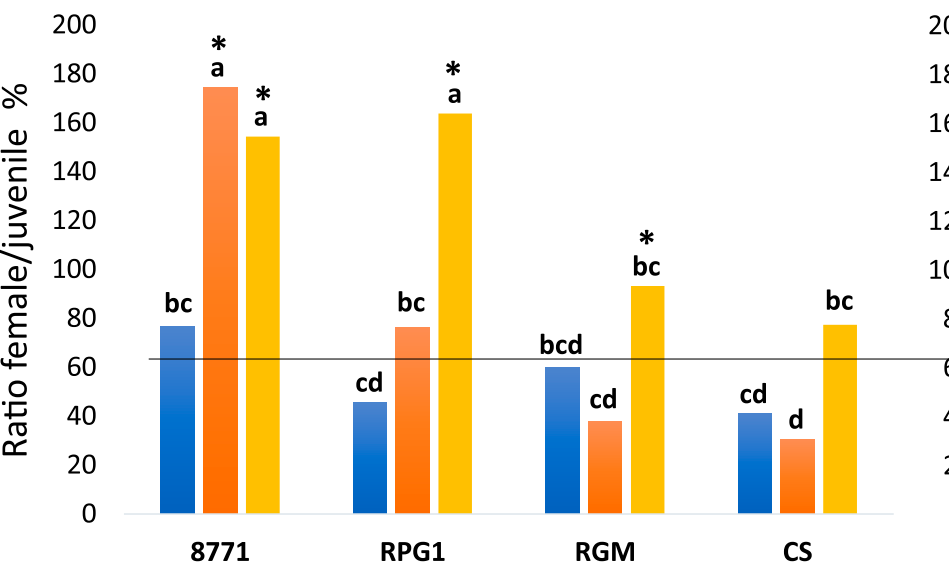

200

180

160

140

120

100

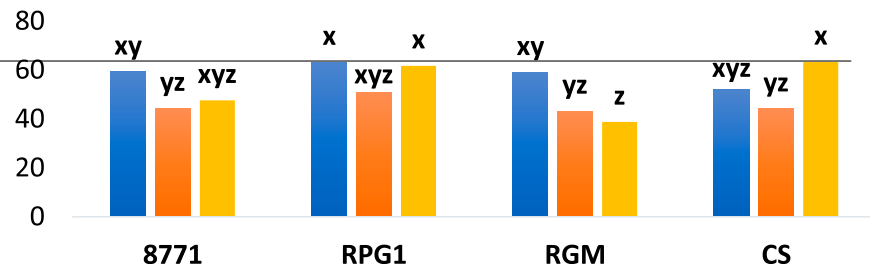

Fig. 5. Effect of the age of the plants from accessions 8771, RPG1, RGM, and CS on the ratio female/juvenile of Xiphinema index after 4 , 5, and 6 years. A, Cuttings = plants obtained from hardwood cuttings. B, In vitro = plants obtained from in vitro. For each propagation type, bars with different letters significantly differ according to Fisher's least significant difference test $(P \leq 0.05)$. The horizontal line highlights the maximal value (63.3\%) in in vitro plants. In cuttings (A), bars with an asterisk are significantly higher $(t$ test, $P \leq 0.05)$ than the corresponding bars (same accession and age) from in vitro (B).

TABLE 1. Effect of the plant propagation type on the frequency of males of Xiphinema index out of the total nematode number in resistant accessions 8771 and RPG1 at 4,5 , and 6 years

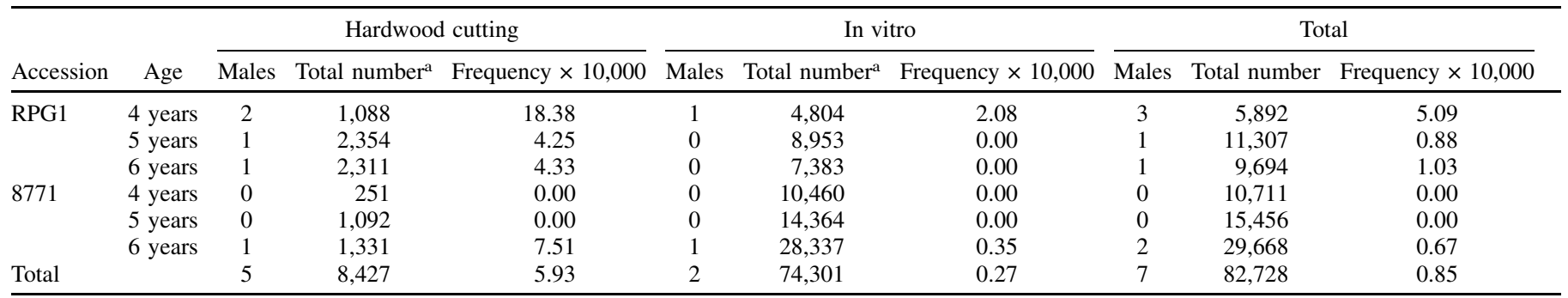

${ }^{a}$ Cumulated number of nematodes counted in all replicates from an accession with a given propagation type at a given age. 


\section{DISCUSSION}

Our experiment monitored over a long duration the host suitability of resistant accessions, together with reference accessions of intermediate and susceptible status, according to the age and the propagation type of the plant. Our results highlight major points that are summarized hereafter.

Resistance is durable in plants obtained from hardwood cuttings but not from in vitro. In hardwood-cutting plants of 8771 and RPG1 accessions, RFs remained lower than 1 (Fig. 3A). The overall significantly higher RF in RPG1 (BC1-level plants) than in 8771 (F1-level plants) (Supplementary Table S3A) agrees with the hypothesis of a dilution of resistance factors in $\mathrm{BC} 1$ compared with $\mathrm{F} 1$ accessions. This result is in line with the fact that all three main QTLs XiR2, XiR3, and XiR4 (Rubio et al. 2020) involved in the resistance of 8771 might not have been inherited in RPG1. The same overall significant difference was observed for nematode numbers per gram of roots (Supplementary Table S3B).

By contrast, in our experiment with an initial in vitro propagation type, we have observed an increase of the RFs and nematode densities on both the $\mathrm{F} 1$ (8771) and $\mathrm{BC} 1$ (RPG1) resistant accessions (Figs. 3B and 4B). At the F1 level, our results on 8771 confirm those previously obtained by Esmenjaud et al. (2010) on the accession VRH 97-99-79 (muscadine R source Carlos) obtained from in vitro and for which an increase of the RF had been observed over 1 to 4 years. At the BC1 level (RPG1), our RF data are also in agreement with those obtained in this previous study. These results with RFs much higher than 1 illustrate a breakdown of resistance. Susceptible (CS) and intermediate (RGM) accessions have shown high RFs that might have reached a maximum at 4 to 6 years. Our results for these accessions are also in line with Esmenjaud et al. (2010) regarding the control susceptible accession $V$. rupestris 'du Lot' and 'RGM'.

Consequently, even though our inoculum was a mix of four lines representative of the nematode diversity, the phenomenon of nematode multiplication over years has not been observed in resistant accessions obtained from hardwood cuttings. We showed that the muscadine-derived resistance strategy based on this propagation type appears durable. No previous comparative evaluations had been performed between plants obtained from hardwood cuttings and plants obtained from in vitro and our results are quite original.

The perennial status of grapevine crops (long interaction between the nematode pest and its host) and the faculty of the meiotic parthenogenetic species $X$. index (Dalmasso 1970) to reproduce sexually (even if it is rare) in the field (Villate et al. 2010) should theoretically increase the risk for resistance breaking consecutive to nematode adaptation (McDonald and Linde 2002). Nevertheless, in our experiment if such an adaptation to resistance factors had occurred, it should have been observed whatever the propagation status. It is not the case as no increase of RFs or nematode densities happened over years in resistant plants obtained from cuttings. Moreover, in plants obtained from in vitro the increase of RFs and nematode densities appears independent from their host suitability status as this phenomenon has been observed for the resistant accessions as well as for the intermediate RGM and susceptible CS accessions. Nevertheless, the definitive confirmation that nematodes reproducing on resistant plants obtained from in vitro are not resistance-adapted individuals would need to save them and then evaluate their reproduction on plants propagated from cuttings in comparison with nematodes that have never been faced to resistant plants.

What might explain the higher nematode numbers in plants of in vitro origin? Our results clearly showed that the previous in vitro culture steps of the plants have influenced their host suitability to $X$. index by increasing the nematode multiplication. In grapevine, the effect of in vitro culture on susceptibility to pests and pathogens has been observed (Nozeran 1978) and linked early (Grenan 1982) to the juvenile characteristics of in vitrocultured plants. In our study, the increased nematode numbers may be at least partly due to the modification of the root architecture in the plants obtained from in vitro that are characterized by root systems with a more compact shape, a delayed lignification and a higher density of rootlets and root apices. Consequently monitoring over years, e.g., the number of rootlets and root apices (where nematodes feed from) per volume of soil in plants of both propagation types would be highly informative.

In culture media, auxin hormones may influence regulation of pathogen resistance responses. Auxins have direct and indirect effects on many processes associated to plant development by regulating cell wall architecture and root morphology (Čarná et al. 2014). They may also make the plant more vulnerable to pathogens by loosening cell walls through the induction of the expression of expansin proteins (Denancé et al. 2013; Ding et al. 2008). Auxin signaling is directly repressed by the basal plant immunity and in particular through the PTI (PAMP-triggered immunity) machinery (Yang et al. 2013). Auxin can also negatively impact plant defense by interfering with other hormone signaling pathways (RobertSeilaniantz et al. 2011; Yang et al. 2013). In our study, the use of the IBA auxin in the rooting medium preceding the experimental set-up in soil might also be involved in the modification of resistance (Supplementary Table S2). Nevertheless, in Prunus accessions carrying the $M a$ gene for resistance to the root-knot nematode M. arenaria (Claverie et al. 2011), IBA used in the in vitro rooting medium did not modify the resistance level 70 days after plant transfer into a sandy soil (Esmenjaud et al. 1993, 1996). Consequently, in this later plant-nematode interaction the putative effect of this auxin hormone on the resistance is labile.

Nevertheless, even though the particular developmental characteristics of the roots in the plants obtained from in vitro are surely the major factor of the increased nematode multiplication in our experiment, other hypotheses at least partly involved in this phenomenon cannot be discarded. In particular, the sterile initial status of both the plant obtained from in vitro and the soil substrate is presumably a feature that might have favored the development of an ectoparasitic species such as $X$. index by reducing the density of competitive soil microorganisms in the rhizosphere and their cognate impact on nematode reproduction. An analysis of the soil microflora harbored by the containers of each propagation type over years would certainly reveal interesting clues. In this later hypothesis, as in that referring to the modification of root characteristics, it remains highly surprising that changes in nematode reproduction are still noticeable after 6 years. Nevertheless, in our experiment the long survival of $X$. index (raised in the next paragraph) is a trait that undeniably makes it more difficult to decipher its fine interaction with resistant plants.

Propagation type affects the structure of the nematode population. The ratio female/juvenile appeared linked to the plant resistance status. In plants showing a low RF, we observed a marked increase of the female proportion. A first hypothesis is that this may be due to the long life duration of the female stage. Actually, in hardwood-cutting plants from the accessions 8771 and RPG1, the populations become older by comparison with plants obtained from in vitro. Indeed, the dagger nematode $X$. index has been shown to survive for more than 4 years, while starving in the soil without plant host (Demangeat et al. 2005). In grapevine plots, the actual survival of adult nematodes is estimated to be 7 years minimum (Vuittenez et al. 1969). At years 5 and 6, survival may explain why a few nematodes are still recovered in our study even in pots containing 6-year-old resistant plants obtained from hardwood cuttings. Nevertheless, to explain the high female proportion in resistant plants, another hypothesis based on a low fertility of newly developed females (i.e., producing few eggs) cannot be excluded and the simultaneous presence of ancient and new females is plausible. The ratio female/juvenile also increased significantly in RGM at 6 years, which may be consecutive to high nematode 
densities in the limited space and/or food resource available from containers with aged plants of this accession.

The rare occurrence of males in resistant plants (mean value 0.85 per 10,000$)$ is in line with the data previously obtained by other authors (Dalmasso 1970; Luc and Cohn 1982; Siddiqi 1986), even though an exception has been reported in Australia with a proportion of males as high as 1 to $2 \%$ (Harris 1977). Our results suggest that male frequencies are higher in plants obtained from hardwood cuttings than from in vitro (Table 1). Considering that the plants of in vitro origin had a weakened resistance, our data would agree with the hypothesis that the higher the resistance, the higher the male occurrence.

\section{ACKNOWLEDGMENTS}

We thank B. Douens, J.-P. Petit, and C. Hévin for their contribution to propagation of hardwood cuttings.

\section{LITERATURE CITED}

Andret-Link, P., Laporte, C., Valat, L., Ritzenthaler, C., Demangeat, G., Vigne, E., et al. 2004. Grapevine fanleaf virus: Still a major threat to the grapevine industry. J. Plant Pathol. 86:183-195.

Boubals, D., and Pistre, R. 1978. Résistance de certaines Vitacées et des portegreffes usuels en viticulture au nématode Xiphinema index et à l'inoculation du court noué (GFV). Pages 200-207 in: Génétique et Amélioration de La Vigne. INRA, Paris.

Bouquet, A., Danglot, Y., Torregrosa, L., Bongiovanni, M., Castagnone-Sereno, P., Esmenjaud, D., and Dalmasso, A. 2000. Breeding rootstocks resistant to grape fanleaf virus spread, using Vitis $\times$ Muscadinia hybridization. Acta Hortic. 528:517-526.

Čarná, M., Repka, V., Skupa, P., and Sturdík, E. 2014. Auxins in defense strategies. Biologia 69:1255-1263.

Claverie, M., Dirlewanger, E., Bosselut, N., Van Ghelder, C., Voisin, R., Kleinhentz, M., Lafargue, B., Abad, P., Rosso, M.-N., Chalhoub, B., and Esmenjaud, D. 2011. The Ma gene for complete-spectrum resistance to Meloidogyne species in Prunus is a TNL with a huge repeated C-terminal post-LRR region. Plant Physiol. 156:779-792.

Dalmasso, A. 1970. Gametogenesis of genus Xiphinema and Longidorus (Nematoda: Dorylaimida). Comptes Rendus Acad. Sci. Serie D. Sci. Nat. 6: 824-827.

Demangeat, G., Voisin, R., Minot, J.-C., Bosselut, N., Fuchs, M., and Esmenjaud, D. 2005. Survival of Xiphinema index in vineyard soil and retention of Grapevine fanleaf virus over extended time in the absence of host plants. Phytopathology 95:1151-1156.

Denancé, N., Sanchez-Vallet, A., Goffner, D., and Molina, A. 2013. Disease resistance or growth: The role of plant hormones in balancing immune responses and fitness costs. Front. Plant Sci. 4:155.

Ding, X., Cao, Y., Huang, L., Zhao, J., Xu, C., Li, X., and Wang, S. 2008. Activation of the indole-3-acetic acid-amido synthetase GH3-8 suppresses expansin expression and promotes salicylate- and jasmonate-independent basal immunity in rice. Plant Cell 20:228-240.

Esmenjaud, D., and Bouquet, A. 2009. Selection and application of resistant germplasm for grapevine nematodes management. Pages 195-214 in: Integrated Management of Fruit Crops Nematodes. Springer, New York.

Esmenjaud, D., Minot, J. C., Voisin, R., Bonnet, A., and Salesses, G. 1996. Inheritance of resistance to the root-knot nematode Meloidogyne arenaria in Myrobalan plum. Theor. Appl. Genet. 92:873-879.

Esmenjaud, D., Minot, J. C., Voisin, R., Salesses, G., Poupet, R., and Onesto, J. P. 1993. Assessment of a method using plantlets grown previously in vitro for studying resistance of Prunus cerasifera HER (Myrobalan plum) to Meloidogyne spp. Nematropica 23:41-48.

Esmenjaud, D., van Ghelder, C., Voisin, R., Bordenave, L., Decroocq, S., Bouquet, A., and Ollat, N. 2010. Host suitability of Vitis and Vitis-Muscadinia material to the nematode Xiphinema index over one to four years. Am. J. Enol. Vitic. 61: 96-101.

Grenan, S. 1982. Quelques réflexions à propos de modifications morphogénétiques consécutives à la culture in vitro chez la vigne. Ann. Sci. Naturelles Bot. Biol. Végétale 4:135-146.

Harris, A. R. 1977. A first record of Tylenchulus semipenetrans and males of Xiphinema index in vineyards in North-eastern Victoria. Australas. Plant Pathol. 6:1-2.
Hooper, D. J. 1986. Extraction of free-living stages from soil. Pages 5-30 in: Laboratory Methods for Work with Plant and Soil Nematodes. J. Southey, ed. Ministry of Agriculture, Fisheries and Food, London.

Hwang, C. F., Xu, K., Hu, R., Zhou, R., Riaz, S., and Walker, M. A. 2010. Cloning and characterization of XiRl, a locus responsible for dagger nematode resistance in grape. Theor. Appl. Genet. 121:789-799.

Jones, J. T., Haegeman, A., Danchin, E. G. J., Gaur, H. S., Helder, J., Jones, M. G. K., et al. 2013. Top 10 plant-parasitic nematodes in molecular plant pathology. Mol. Plant Pathol. 14:946-961.

Luc, M., and Cohn, E. 1982. The male of Xiphinema index Thorne \& Allen, 1950 (Nematoda: Longidoridae). Rev. Nematol. 5:211-215.

McDonald, B. A., and Linde, C. 2002. Pathogen population genetics, evolutionary potential, and durable resistance. Annu. Rev. Phytopathol. 40: 349-379.

Murashige, T., and Skoog, F. 1962. A revised medium for rapid growth and bio assays with tobacco tissue cultures. Physiol. Plant. 15:473-497.

Nguyen, V. C. 2018. Genetic diversity of the grapevine vector nematode Xiphinema index and application to optimize the resistance strategy. Ph.D. thesis. Doctoral School SVS. Université Côte d'Azur, Nice, France.

Nguyen, V. C., Villate, L., Gutierrez-Gutierrez, C., Castillo, P., Van Ghelder, C., Plantard, O., and Esmenjaud, D. 2019. Phylogeography of the soil-borne vector nematode Xiphinema index highly suggests Eastern origin and dissemination with domesticated grapevine. Sci. Rep. 9:7313.

Noe, J. P. 1985. Analysis and interpretation of data from nematological experiments. Pages 187-197 in: An Advanced Treatise on Meloidogyne. K. R. Barker, C. C. Carter, and J. N. Sasser, eds. Department of Plant Pathology, North Carolina State University.

Nozeran, R. 1978. Polymorphisme des individus issus de la multiplication végétative des végétaux supérieurs, avec conservation du potentiel génétique. Physiol. Veg. 16:177-194.

Nozeran, R. 1980. La multiplication végétative chez les végétaux supérieurs. Pages 1-29 in: La Multiplication Végétative des Plantes Supérieures. Gauthiers-Villars, Paris.

Ollat, N., Fernandez, L., Romieu, C., Duchene, E., Lissarague, J. R., Lecourieux, D., et al. 2011. Multidisciplinary research to select new cultivars adapted to climate changes. 17th International Symposium of GiESCO, Asti-Alba, Italy.

Péros, J., Bonnel, E., Roques, D., and Paulet, F. 1994. Effect of in vitro culture on rust resistance and yield in sugarcane. Field Crops Res. 37:113-119.

Robert-Seilaniantz, A., MacLean, D., Jikumaru, Y., Hill, L., Yamaguchi, S., Kamiya, Y., and Jones, J. D. G. 2011. The microRNA miR393 re-directs secondary metabolite biosynthesis away from camalexin and towards glucosinolates. Plant J. 67:218-231.

Rubio, B., Lalanne-Tisné, G., Voisin, R., Tandonnet, J.-P., Portier, U., Van Ghelder, C., et al. 2020. Characterization of genetic determinants of the resistance to phylloxera, Daktulosphaira vitifoliae, and the dagger nematode Xiphinema index from muscadine background. BMC Plant Biol. 20: 213.

Siddiqi, M. R. 1986. Xiphinema index. Page Set 3, No 45 in: C.I.H. Descriptions of Plant Parasitic Nematodes. Commonwealth Institute of Parasitology, CAB International, Wallingford, U.K.

Van Ghelder, C., Reid, A., Kenyon, D., and Esmenjaud, D. 2015. Detection of nepovirus vector and nonvector Xiphinema species in grapevine. Plant Pathology: Methods in Molecular Biology. C. Lacomme, ed. Vol. 1302. Humana Press, New York.

Villate, L. 2008. Origine, variabilité et gestion des populations de Xiphinema index, le nématode vecteur du Grapevine fanleaf virus (GFLV). Ph.D. thesis. Doctoral School VAS, Rennes, ENSA, France.

Villate, L., Esmenjaud, D., Coedel, S., and Plantard, O. 2009. Development of nine polymorphic microsatellite markers for the phytoparasitic nematode Xiphinema index, the vector of the Grapevine fanleaf virus. Mol. Ecol. Resour. 9:229-232.

Villate, L., Esmenjaud, D., Van Helden, M., Stoeckel, S., and Plantard, O. 2010. Genetic signature of amphimixis allows for the detection and fine scale localization of sexual reproduction events in a mainly parthenogenetic nematode. Mol. Ecol. 19:856-873.

Villate, L., Morin, E., Demangeat, G., van Helden, M., and Esmenjaud, D. 2012. Control of Xiphinema index populations by fallow plants under greenhouse and field conditions. Phytopathology 102:627-634.

Vuittenez, A., Legin, R., and Kuszala, J. 1969. Les viroses de la vigne. Pages 557-578 in: Journées Françaises d'Etudes et d'Information. ACTA Editions, Paris.

Yang, D.-L., Yang, Y., and He, Z. 2013. Roles of plant hormones and their interplay in rice immunity. Mol. Plant 6:675-685. 